\title{
Axis I disorders are common in people with severe borderline personality disorder, but decrease with time
}

Zanarini MC, Frankenburg FR, Hennen J, et al. Axis I comorbidity in patients with borderline personality disorder: 6-year follow-up and prediction of time to remission. Am J Psychiatry 2004; 161:2108-14.

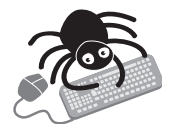

This article contains extra text on the EBMH website

What is the prevalence of axis I comorbidities in people with severe borderline personality disorders and how do they affect prognosis?

\section{METHODS}

$\square$

Design: Prospective cohort study.

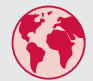

Setting: McLean Hospital, MA, USA; enrolment 1992-1995.

임의

Population: 362 adult inpatients (female 77\%; Caucasian $87 \%$ age 18-35 years (mean 27 years)) with DSM-III-R and Revised Diagnostic Interview for Borderlines (DIB-R) diagnosis of borderline personality disorder (BPD) $(n=290)$ or other axis II disorder ( $n=72$ ). Exclusions: non-English speaking; $I Q<70$; current or prior schizophrenia, schizoaffective disorder, bipolar I disorder, or an organic condition causing psychiatric symptoms.

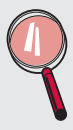

Assessment: Three semistructured interviews at baseline and 2 year intervals to assess participants for borderline personality, axis I, and axis II disorders (Structured Clinical Interview for DSMIII-R Axis I disorders; DIB-R; Diagnostic Interview for DSM-III-R Personality Disorders). Random effects regression models were used to analyse prevalence of 20 axis I disorders. Discrete time to event modelling methods were used to calculate hazard ratios and $95 \% \mathrm{Cl}$ for time to remission.

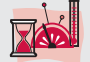

Outcomes: Presence of axis I disorders (DSM-III-R), time to remission.

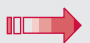

Follow up period: 6 years.

\section{MAIN RESULTS}

Axis I disorders were common among inpatients with BPD (see http://www.ebmentalhealth.com/supplemental for table). Mood and anxiety disorders were significantly more common in severe BPD than in other axis II disorders $(\mathrm{p}<0.002$; see table). The overall prevalence of axis I disorders decreased over time, and was more pronounced among people in remission. People without substance use disorders, post-traumatic stress disorder (PTSD), mood disorders, and eating disorders had earlier remission than those with these disorders (substance use disorders: HR 4.0, 95\% CI 2.6 to 6.2; PTSD: HR 2.7, 95\% CI 2.0 to 3.8; mood disorders: HR 2.0, 95\% CI 1.4 to 2.9 ; eating disorders: HR $1.5,95 \%$ CI 1.1 to 2.1 ).

\section{CONCLUSIONS}

Over time, axis I disorders decrease in people with severe BPD, particularly if they experience remission. Absence of comorbid substance use disorders is associated with earlier remission, suggesting that these significantly hinder recovery from BPD.

\section{NOTES}

As participants were very disturbed inpatients, the findings may not apply to less severely disturbed people with BPD. The temporal relationship between comorbidity and remission was not clearly established, therefore a causality cannot be imputed.

For correspondence: Dr Mary C Zanarini, McLean Hospital, 115 Mill Street, Belmont, MA 02478, USA; zanarini@mclean.harvard.edu

Sources of funding: National Institute of Mental Health, USA.

\section{Commentary}

7 anarini et al's paper is the latest of a series based on a landmark 6 year naturalistic prospective study of borderline personality - disorder (BPD). ${ }^{1}$ Other studies that have assessed the long term outcome of $\mathrm{BPD}^{2} 3$ have not used the same rigor of design. Participants in this study were derived from people hospitalised at McLean, a private psychiatric hospital; 290 people with BPD were compared with 72 with other personality disorders. The major findings and implications of this study are:

(1) Within 6 years, BPD criteria had remitted for most participants, although most continued to receive psychotherapy and pharmacotherapy, ${ }^{4}$ implying that BPD has a relatively good prognosis with appropriate treatment.

(2) Relapse rates were low, suggesting that BPD is not simply a variant of bipolar disorder, which would be expected to have a high relapse rate.

(3) Multiple axis I comorbidities were common. Most people with BPD had both a mood and an anxiety disorder. Substance use and eating disorders were also common. The presence of major axis I pathology may partially account for why BPD is so often under-diagnosed.

(4) Many people with BPD continued to suffer significant axis I pathology despite multimodal treatment, indicating that axis I disorders may not respond to usual treatments when comorbid BPD is present. ${ }^{5}$

(5) Remission of axis I disorders was highly correlated with remission of BPD; this may indicate that specific treatment for BPD is necessary for successful remission of comorbid axis I disorders.

(6) Comorbid substance use disorder was a strong negative prognostic factor, consistent with other studies suggesting that people who have both disorders may have a worse outcome. ${ }^{36}$ Most published randomised treatment studies of BPD have excluded subjects with substance use disorders. The development of effective treatments for this population should be an area for future investigation.

The major limitation is the selection of participants-inclusion of only inpatients selects for greater axis I comorbidity. Most participants were white and had a middle or upper socioeconomic status. Outpatient treatment in the McLean area is probably more readily available and of higher quality because of multiple training institutions in the region. The prognosis of people receiving treatment in the public sector or where specific treatments are unavailable may not be as favourable.

Robert J Gregory, MD SUNY Upstate Medical University, NY, USA

1 Zanarini MC, Frankenburg FR, Hennen J, et al. The longitudinal course of borderline psychopathology: 6-year prospective follow-up of the phenomenology of borderline personality disorder. Am J Psychiatry 2003;160:274-83.

2 McGlashan TH. The Chestnut Lodge follow-up study, III: long-term outcome of borderline personalities. Arch Gen Psychiatry 1986;43:20-30.

3 Stone MH. The Fate of Borderline Patients. New York: Guilford, 1990

4 Zanarini MC, Frankenburg FR, Hennen J, et al. Mental health service utilization by borderline personality disorder patients and axis II comparison subjects followed prospectively for six years. J Clin Psychiatry 2004;65:28-36.

5 Feske U, Mulsant BH, Pilkonis PA, et al. Clinical outcome of ECT in patients with major depression and comorbid borderline personality disorder. Am J Psychiatry 2004;161:2073-80.

6 Ryle A, Golynkina K. Effectiveness of time-limited cognitive analytic therapy of borderline personality disorder: factors associated with outcome. $\mathrm{Br} J$ Med Psychol 2000;73:197-210. 